\title{
Evidence for Shocks and Increased SFE in the Lyman Alpha Reference Sample
}

\author{
Johannes Puschnig, Matthew Hayes and Göran Östlin \\ Department of Astronomy, Oskar Klein Centre, Stockholm University \\ email: johannes.puschnig@astro.su.se
}

We report on first molecular gas observations in the Lyman Alpha Reference Sample (LARS; Hayes et al. 2013, 2014, Östlin et al. 2014), which were performed using the 45m telescope at the Nobeyama Radio Observatory (NRO). The beamsize at the observed ${ }^{12} \mathrm{CO}(1-0)$ emission is $\approx 15^{\prime \prime}$ corresponding to $\approx 12 k p c$ at the given redshift of $z \approx 0.04$. We detected strong ${ }^{12} \mathrm{CO}$ emission in LARS 3 (Arp 238), marginally detected LARS 8 (SDSS 1250+0734) and derived an upper limit for LARS 9 (IRAS 08208+2816).

Our data analysis reveals that LARS 8 and 9 are critically offset from the Kennicutt-Schmidt law leading to an increase in star formation efficiency (SFE). We note that the metallicity in the presented galaxies is sub-solar with a value of $[12+\log (\mathrm{O} / \mathrm{H})] \approx 8.4$. Thus, the applicability of $\mathrm{CO}$ as a molecular gas tracer is limited and using a constant Milky Way conversion factor $\alpha_{C O}=4.3$ is most likely not adequate (see green markers in

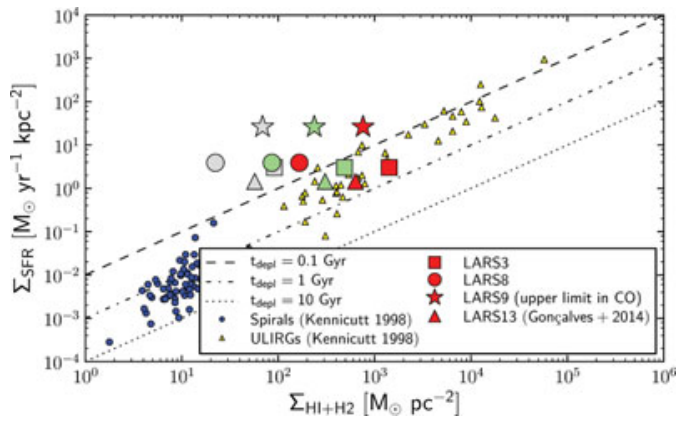
fig.). Therefore, we also make use of a metallicty-dependent $\alpha_{C O}$ which was calculated based on the findings of Magdis et al. (2011) and relies on the quite tight correlation between the gas-to-dust ratio and the metallicity. However, even after applying this Z-dependent term, the SFE remains offset by a factor of $\approx 5$ (see red markers in fig.) compared to star forming disks.

We interpret the enhanced SFE as a result of shock heating in the ISM introduced by tidal forces. The external ISM pressure exceeding the dynamical pressure of molecular clouds, triggers their collapse and subsequent fragmentation. As shown by Zubovas et al. (2014), in such environments of high pressure, new stars preferentially form in massive and compact clusters located in the centre of the initial cloud rather than along cloud filaments. This is supported by our HST images which show tidal features as a result of merging processes and the existence of dense and supermassive stellar associations.

In the case of LARS 09, this scenario is supported by our Herschel/PACS spectroscopy, because the observed [CII] $158 \mu \mathrm{m}$ line is too strong to be explained by cooling in photodissociation regions (PDRs) from star formation only. Most likely, the galaxy contains large amounts of shock-heated gas undergoing turbulent energy dissipation.

In summary, there is evidence for an increased SFE due to shock heating in some LARS galaxies, but uncertainties due to the conversion factor can still affect the result. In a next step, we will resolve the issue by probing higher $\mathrm{CO}$ transitions and other molecular lines, which then enable us to compar e the observations to PDR models in order to constrain the underlying physical conditions. 


\section{References}

Hayes, M., Östlin, Duval, F., et al. 2014, ApJ, 782, 6

Magdis, G. E., Daddi, E., Elbaz, D., et al. 2011, ApJ, 740, 15

Zubovas, K., Sabulis, K., Naujalis, R. 2014 MNRAS, 442, 2837

Hayes, M., Östlin, G., Schaerer, D., et al. 2013, ApJL, 765, L27

Östlin, G., Hayes, M., Duval, F., et al. 2014, ApJ, in prep. 